\title{
Manipulation of the spontaneous emission dynamics of quantum dots in two-dimensional photonic crystals
}

\author{
A. Kress, * F. Hofbauer, N. Reinelt, M. Kaniber, H. J. Krenner, R. Meyer, G. Böhm, and J. J. Finley \\ Walter Schottky Institut and Physik Department, Technische Universität München, Am Coulombwall 3, D-85748 Garching, Germany
}

(Received 22 December 2004; revised manuscript received 1 April 2005; published 14 June 2005)

\begin{abstract}
We demonstrate the ability to control the spontaneous emission dynamics of self-assembled quantum dots via the local density of optical modes in two-dimensional (2D) photonic crystals. We show that an incomplete 2D photonic band gap is sufficient to significantly lengthen the spontaneous emission lifetime $(>2 \times)$ over a wide bandwidth $(\Delta \lambda \geqslant 40 \mathrm{~nm})$. For dots that are both spectrally and spatially coupled to strongly localized $\left[V_{\text {modes }} \sim 1.5(\lambda / n)^{3}\right]$, high $Q \sim 2700$ optical modes, we have directly measured a strong Purcell-enhanced shortening of the emission lifetime $\geqslant 5.6 \times$, limited only by our temporal resolution. Analysis of the spectral dependence of the recombination dynamics shows a maximum lifetime shortening of $19 \pm 4$. From the directly measured enhancement and suppression we show that the single-mode coupling efficiency for quantum dots in such structures is at least $\beta=92 \%$ and is estimated to be as large as $\sim 97 \%$.
\end{abstract}

DOI: $10.1103 /$ PhysRevB.71.241304

PACS number(s): 42.70.Qs, 73.21.La, 78.67.Hc, 42.50.Pq

Designer photonic materials fabricated from periodic dielectrics provide a direct route toward achieving complete control of the spontaneous emission of solids. ${ }^{1,2}$ The ability to manipulate the strength of the light-matter coupling in this way lies at the very heart of modern optics, with a variety of potential applications ranging from integrated photonics ${ }^{3}$ to fundamental quantum optics. ${ }^{4-7}$ One of the most remarkable quantum optical phenomena is the deterministic generation of single photons from isolated quantum emitters. In this context, individual semiconductor quantum dots (QDs) are ideal solid-state emitters due to their high radiative efficiency, stability, and ease of incorporation into active devices. ${ }^{8-11}$ However, for QDs embedded within an isotropic semiconductor the single-photon extraction efficiency is extremely low $\left(\eta_{e x}<1 \%\right)$ limiting their realistic potential for applications in quantum information science., 5 This problem can be addressed by locating QDs within optical cavities and utilizing the Purcell effect ${ }^{13}$ to funnel single photons into a single optical mode for collection. ${ }^{10}$ Using such approaches, $\eta_{e x}$ larger than a few percent have been reported for single dots incorporated into pillar microcavities. ${ }^{10}$ Cavities realized using photonic crystals (PCs) may provide maximum flexibility to tune the local density of photon states over a much wider bandwidth and achieve full control of the spontaneous emission via the strength of the local vacuum field fluctuations. ${ }^{2}$ Furthermore, strongly localized modes in PCs combine a planar geometry with high-quality factors $\left(Q=\omega \tau_{\text {photon }}\right)$ and small effective mode volume $\left(V_{\text {eff }}\right)$ (Refs. 15-17), potentially advantageous properties for achieving strong Purcell enhancement and realizing efficient QD-based single-photon emitters.

In this paper we demonstrate control of the QD spontaneous emission dynamics in such two-dimensional (2D) PCs. For dots that are both spectrally and spatially coupled to strongly localized high $Q$ cavity modes we directly measure a pronounced shortening of the emission lifetime $(\geqslant 5.6 \times)$, limited only by the temporal resolution of our detection system. Analysis of the spectral dependence of the decay rate as a function of emitter-cavity detuning shows that the maxi- mum enhancement is as large as $19 \pm 4$. A strong $(>2 \times)$ reduction of the emission decay time is observed over a wide bandwidth for dots detuned from the cavity modes, demonstrating that a partial, TE band gap is sufficient to tailor the QD spontaneous emission dynamics. From the directly measured enhancement and suppression of the spontaneous emission lifetime we extract a single-mode coupling efficiency for dots in these PCs of $\beta=92 \%$, the spectrally dependent measurements indicating that it may become as large as $\beta$ $\sim 97 \%$.

The samples investigated consist of a $d=400$-nm-thick Air-GaAs-Air slab waveguide into which a 2D PC is defined by fabricating a triangular lattice of air holes in the GaAs waveguide and defining a suspended membrane by removing an underlying AlAs layer using wet chemical etching. A single layer of nominally $\operatorname{In}_{0.5} \mathrm{Ga}_{0.5} \mathrm{As}$ QDs was incorporated into the center of the GaAs waveguide core as an internal light source. Low-mode volume nanocavities were formed by introducing single missing hole point defects in the hexagonal lattice of holes, realizing $H 1$ resonators. The PCs have a periodicity of $a=300 \mathrm{~nm}$ and the air hole radius $(r)$ was varied to tune the cavity-mode energies through the inhomogeneously broadened spectrum of QD ground states and control the width of the photonic band gap. From a QD areal density of $\sim 200 \mu \mathrm{m}^{-2}$ and the detection spot size of $\sim 1 \mu \mathrm{m}^{2}$ we estimate that only a small number of dots $(\sim 5)$ are spectrally coupled to the high $Q$ cavity modes.

Spatially resolved optical measurements were performed at $T=10 \mathrm{~K}$ using a confocal microphotoluminescence $(\mu \mathrm{PL})$ system that provides a spatial resolution $\sim 1 \mu \mathrm{m}$. For $\mathrm{cw}$ measurements, the samples were excited using a HeNe laser and the resulting PL signal was dispersed by a $0.55-\mathrm{m}$ imaging monochromator and detected using a nitrogen-cooled $\mathrm{Si}$ charged-coupled device (CCD) camera. Time-resolved measurements were performed by exciting the sample using $\sim 50$ ps duration pulses at $\lambda=658 \mathrm{~nm}$ and detecting the temporal decay of the resulting luminescence using a singlephoton Si-avalanche photodetector and time-correlated photon counting electronics. The maximum temporal resolution 

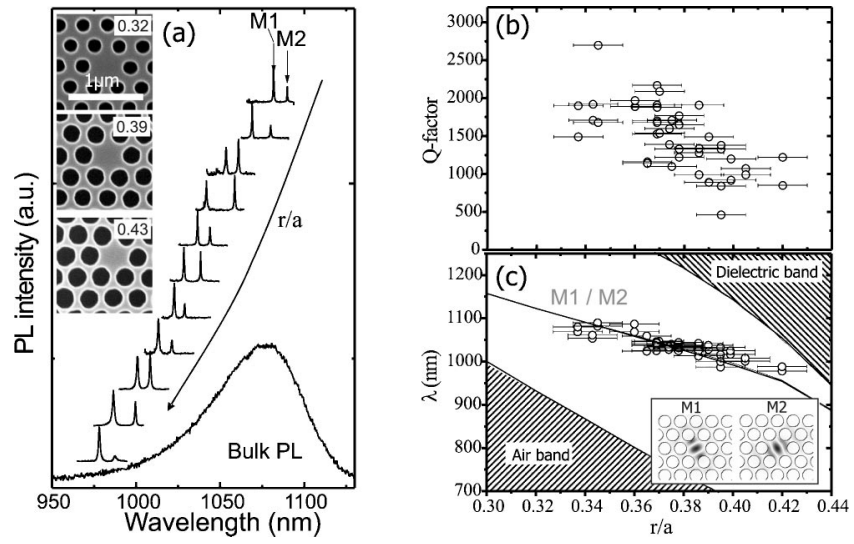

FIG. 1. (a) PL spectra recorded from a series of $H 1$ PC cavities as a function of the ratio of hole radius $r$ to periodicity $a$. [inset of (a)] The SEM images show typical cavities. The ensemble PL is shown for comparison. (b) Measured $Q$ factors for the cavity modes of $>40$ cavities as a function of $r / a$. (c) Calculations of the TEpolarized three-dimensional (3D) band structure with $a=300 \mathrm{~nm}$ showing the localized dipolelike modes $M 1$ and $M 2$ inside the photonic band gap and the experimental data. [inset of (b)] Calculated electric-field profile of the cavity modes.

provided by this system is $\sim 150 \mathrm{ps}$ after deconvolution with the system response function, much shorter than the intrinsic ground-state radiative lifetime of our QDs $\left(\tau_{0} \sim 0.8 \mathrm{~ns}\right)$.

Figure 1(a) compares an ensemble PL spectrum with $\mu \mathrm{PL}$ spectra recorded from a series of $H 1$ cavities as $r / a$ is increased systematically from 0.32 to 0.42 . Over this range of parameters, the $\mu \mathrm{PL}$ spectra reveal a prominent doublet, labeled $M 1$ and $M 2$ in Fig. 1, corresponding to dipolelike cavity modes orientated along the $\Gamma-M$ and $\Gamma-K$ crystal directions [see inset of Fig. 1(c)]. Under the present strong excitation conditions $\left(P_{e x} \sim 100 \mathrm{~W} \mathrm{~cm}^{-2}\right)$ the PL intensity is determined by the QD spontaneous emission lifetime and a $50 \times$ enhancement of the PL intensity is observed for dots spectrally on resonance with the cavity modes when compared with dots that are detuned. This observation indicates the presence of pronounced cavity QED effects, an expectation confirmed by our time-resolved measurements presented below.

Figure 1(b) shows the cavity-mode $Q$ factors deduced for over 40 structures plotted as a function of $r / a$. A significant increase of $Q$ from $\sim 500$ to $\sim 2700$ is observed as $r / a$ is reduced from 0.42 to 0.33 . This can be explained by considering the position of the cavity modes within the TE photonic band gap. ${ }^{16}$ Figure 1(c) shows the calculated band structure for our structures as a function of $r / a .{ }^{18}$ The continuum $d i$ electric and air bands are marked by the shaded regions, together with the TE photonic band gap and the $M 1-M 2$ doublet (solid lines). ${ }^{19}$ The calculated wavelength of the cavity modes and its dependence on $r / a$ are in good quantitative agreement with our measurements, confirming their identification. ${ }^{20}$ The calculations presented in Fig. 1(c) show that $M 1$ and $M 2$ shift progressively deeper into the photonic band gap as $r / a$ is reduced. As a consequence, the modes couple more weakly to the dielectric band continuum resulting in the observed enhancement of the $Q$ factor. ${ }^{16}$ We now

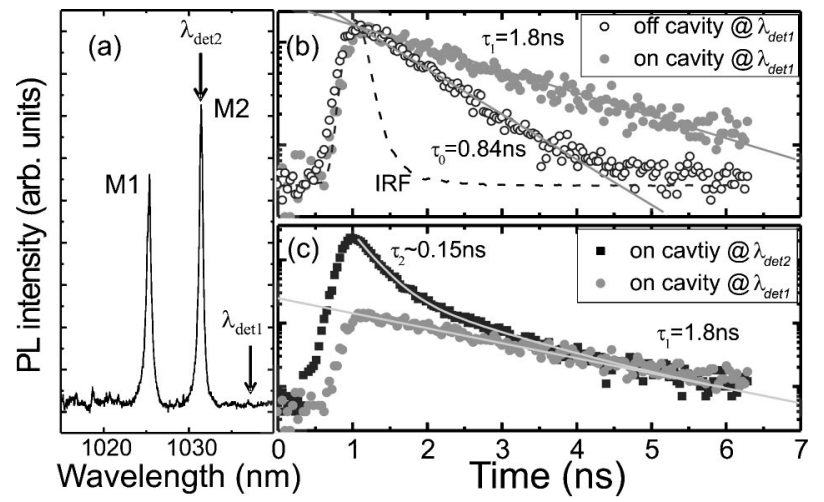

FIG. 2. (a) PL spectrum of the selected cavity. (b) Comparison of decay transients recorded from QDs detuned from the cavity modes $\left(\lambda_{\text {det } 1}=1037 \mathrm{~nm}\right)$ either (i) within the unpatterned GaAs membrane $\left(\tau_{0}\right.$-open circles) or (ii) from the PC $\left(\tau_{1}\right.$-filled circles). The dashed line is the instrument response function (IRF) of our setup. (c) Decay transients recorded from the PC $H 1$ cavity both in resonance with $M 2$ at $\lambda_{d e t 2}=1031.5 \mathrm{~nm}$ (filled squares) and detuned at $\lambda_{\text {det } 1}=1037 \mathrm{~nm}$ (filled circles).

shift our attention to the emission dynamics of QDs whose emission frequency lies throughout the TE photonic band gap, both in and out of resonance with the highest $Q$ cavity modes.

The maximum photon lifetime in our cavities is $\tau_{\text {photon }}$ $=Q_{\max } / \omega \sim 2 \mathrm{ps}$, much shorter than the typical QD spontaneous emission lifetime $\left(\tau_{0} \sim 0.8 \mathrm{~ns}\right)$. Furthermore, since the QD homogeneous linewidth is much narrower than the cavity mode $\left[\Delta \lambda_{c}=\lambda_{c} / Q \sim 0.5 \mathrm{~nm}\right.$, c.f. $\Delta \lambda_{Q D} \ll 0.1 \mathrm{~nm}$ (Ref. 21)] the light-matter coupling remains in the perturbative regime and can be described by the Purcell effect. ${ }^{13,14}$ In this case, for an ideal emitter on resonance with the cavity mode the spontaneous decay lifetime is reduced by a factor $F_{p}$ $=3 Q /\left(4 \pi^{2} V_{\text {mode }}\right)$, where $V_{\text {mode }}$ is the effective volume of the cavity mode in units of $\left(\lambda_{c} / n\right)^{3}$. For the cavities discussed here, we calculate $F_{p} \sim 100$, for $Q \sim 2000$ and $V_{\text {mode }}$ $\sim 1.5\left(\lambda_{c} / n\right)^{3}$, in good accordance with the $\sim 50 \times$ total enhancement of the emission intensity observed for QDs spectrally in resonance with the cavity modes [see Fig. 1(a)]. However, to unambiguously separate the influence on the QD radiative lifetime of the local density of photonic states from simple improvements of the collection efficiency due to the PC, we performed time-resolved measurements.

A PL spectrum from the cavity selected for time-resolved studies is presented in Fig. 2(a), showing cavity modes suitable for detection using our silicon-based detection system $\left(\lambda_{M 1}=1025.4 \mathrm{~nm}\right.$ with $Q_{M 1}=1500$ and $\lambda_{M 2}=1031.5 \mathrm{~nm}$ with $\left.Q_{M 2}=1950\right)$. We compared $\mu \mathrm{PL}$ decay transients recorded both in and out of resonance with the cavity modes with the intrinsic QD dynamics measured on the unpatterned GaAs membrane without the PC $\left(\tau_{0}\right)$. Figure 2(b) compares raw time-resolved data recorded from QDs in the cavity, but strongly detuned from the cavity mode (filled circles) with reference data recorded at the same wavelength $\left(\lambda_{\operatorname{det} 1}\right.$ $=1037 \mathrm{~nm}$ ) from the unpatterned membrane (open circles). For both transients, we observe monoexponential decays with time constants of $\tau_{1}=1.8 \pm 0.1 \mathrm{~ns}$ and $\tau_{0}$ 


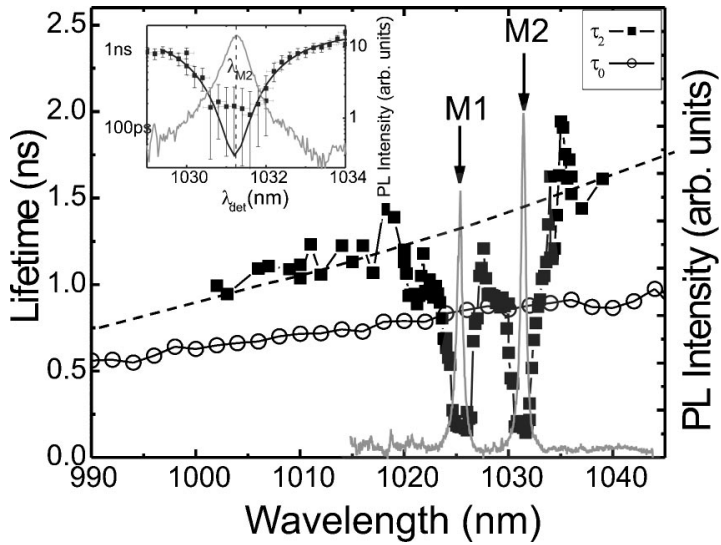

FIG. 3. Spectral dependence of the decay lifetimes for dots within the PC (filled squares) and within the unpatterned membrane (open circles). The dotted line represents a guide to the eye showing the stronger suppression of the spontaneous emission for wavelengths closer to $\lambda_{\text {mid }}^{T E}$. (inset) Fit of Eq. (1) to the spectral dependence of the decay lifetime.

$=0.84 \pm 0.05 \mathrm{~ns}$, respectively. The QDs located within the cavity have much longer decay times $\left(\tau_{1} / \tau_{0} \sim 2\right)$ when compared with dots in the pure membrane, indicating the presence of a gap in the local photonic density of states due to the 2D photonic band gap. ${ }^{2}$ This suggestion is further substantiated by our spectrally resolved measurements presented below.

Figure 2(c) compares a decay transient recorded from dots in the cavity, here recorded in resonance with $M 2$ (filled squares), with the data of Fig. 2(b) detuned strongly by $\sim 5 \mathrm{~nm}$ to longer wavelength (filled circles). In contrast with all dynamics discussed until now, the decay transient recorded on resonance with $M 2$ shows a clear double exponential decay, as confirmed by a fit of $I(t)=A \exp \left(-t / \tau_{2}\right)$ $+B \exp \left(-t / \tau_{1}\right)$ shown on the figure. The longer time constant $\left(\tau_{1}=1.8 \pm 0.1 \mathrm{~ns}\right)$ is identical to that discussed above for QDs spectrally detuned from the cavity mode, whereas the faster transient $\left(\tau_{2} \sim 0.15 \mathrm{~ns}\right)$ is limited by the time resolution of our setup. We identify this behavior as arising from a strong Purcell-enhanced shortening of the emission time, compared with $\tau_{0}$, measured for dots that are both spectrally and spatially on resonance with the cavity mode. From Figs. 2(b) and $2(\mathrm{c})$ we obtain already a factor $\tau_{0} / \tau_{2} \geqslant 5.6 \pm 0.3$, while the decay time $\tau_{2}$ is limited by the system time resolution [see instrument response function in Fig. 2(b)]. The longer decay transient $\tau_{1}$ is identified as arising from QDs that are spectrally on resonance with $M 2$ but do not couple to the cavity mode due to their position outside the cavity, in the body of the PC. From these directly measured decay times for coupled $\left(\tau_{2}\right)$ and uncoupled $\left(\tau_{1}\right)$ dots (see Fig. 3) we obtain ratios of $\tau_{1} / \tau_{2}=12 \pm 1$, defining a single-mode coupling efficiency $\beta=1-\left(\tau_{2} / \tau_{1}\right) \sim 92 \%$ for dots placed both spectrally and spatially on resonance with the cavity mode. This figure of merit provides significant promise for the realization of efficient, QD-based, single-photon sources based on PC cavities. $3,10,11$

Figure 3 compares the spectral dependence of the QD decay time in the membrane, but away from the PC
( $\tau_{0}$ - open circles) and the dominant decay time for dots in the cavity (filled squares), representing the faster of the two time constants extracted from the biexponential fit. A reference PL spectrum is also presented for comparison. The intrinsic QD lifetime $\tau_{0}$ does not vary between QDs in the unprocessed material and in the unpatterned GaAs membrane. It increases weakly from $\tau_{0} \sim 0.65$ to $0.90 \mathrm{~ns}$ as the detection wavelength increases from 1000 to $1040 \mathrm{~nm}$. From the data presented in Fig. 3 the significant lengthening (off resonance) and shortening (on resonance) of the decay liftime discussed above can be clearly observed. Moreover, the lengthening is found to occur over a remarkably wide bandwidth $(\Delta \lambda \geqslant 40 \mathrm{~nm})$ and becomes more pronounced towards longer wavelength as shown schematically by the dashed line in Fig. 3 that acts as a guide to the eye. For the presently investigated PCs, the middle of the TE-polarized photonic band gap lies at $\lambda_{\text {mid }}^{T E} \sim 1100 \mathrm{~nm}$ [see Fig. 1(c)] and there is no overlapping gap for both TE- and TM-polarized waveguide modes. The observed spectral dependence is attributed to a progressive reduction of the local photon density of states as the wavelength approaches $\lambda_{\text {mid }}^{T E}$, indicating that even a partial 2D photonic band gap is sufficient to significantly inhibit spontaneous emission. ${ }^{21}$ We believe that this is due to the predominantly heavy-hole character of the QD ground-state exciton transition $^{22}$ that gives rise to TEpolarized emission. Therefore, we suggest that tailoring of only the TE-optical modes is sufficient to strongly modify the spontaneous emission properties of self-assembled QDs in 2D-PC nanocavities.

The minima in the spectral dependence of the decay lifetime $[\tau(\lambda)]$ close to $M 1$ and $M 2$ are $\sim 4 \times$ broader than the cavity modes in the emission spectrum. Since the Lorentzian cavity modes should lead to a similar spectral profile in $\tau(\lambda)$, this observation indicates that the reduction of the decay time for zero detuning is much larger than the measured $\sim 150 \mathrm{ps,}$ limited by our temporal resolution. In the weak-coupling regime photon reabsorption can be neglected and Fermi's golden rule provides the spontaneous decay time relative to its value in a homogeneous medium $\tau_{0} / \tau_{2}$. (Ref. 23),

$$
\frac{\tau_{0}}{\tau_{2}}=\frac{1}{3} F_{P} \frac{|\vec{E}(\vec{r})|^{2}}{\left|\vec{E}_{\text {max }}\right|^{2}} \frac{\Delta \lambda_{c a v}^{2}}{\Delta \lambda_{c a v}^{2}+4\left(\lambda_{c a v}-\lambda_{Q D}\right)^{2}}+\alpha .
$$

In Eq. (1) $\lambda_{Q D}$ and $\lambda_{c a v}$ are the QD and cavity wavelength and $\Delta \lambda_{\text {cav }}$ is the linewidth of the cavity mode measured from the PL spectrum. Two different decay channels are taken into account in Eq. (1); the first term describes the spontaneous emission of a dot located at $\vec{r}$ into the cavity mode with a local electric field $\vec{E}(\vec{r})$ and a maximum amplitude $\vec{E}_{\text {max }}$, whereas in the second term $\alpha$ describes a possible decay channel due to emission into residual modes in the quasiphotonic band gap. By fitting Eq. (1) to the measured $\tau(\lambda)$ data we extract the decay time on resonance, the best fit is compared with the PL intensity in Fig. 3 (inset) on a logarithmic scale. The fitted spectral dependence of the decay time, now has exactly the same line shape as the PL intensity but with a much shorter decay time $\tau_{2}=44 \pm 8$ ps on resonance. From the fit, we estimate a maximum shortening of the decay time 
by a factor $\tau_{0} / \tau_{2}=19 \pm 4$ for ideally located QDs on resonance, corresponding to a maximum Purcell factor $F_{P}$ $=56 \pm 10$. This value is in fairly good agreement with the maximum value of $\sim 100$ calculated from the measured $Q$ and $V_{e f f}$, the discrepancy probably arising from a displacement of the QDs probed relative to the electric-field antinode in the cavity. For the obtained values of $\tau_{1}=1.8 \mathrm{~ns}$ and $\tau_{2}$ $\sim 50$ ps we estimate a maximum single-mode coupling efficiency for this system of $\beta \sim 97 \%$.

In summary, we have investigated the influence of the modified density of states in PC nanocavities on the spontaneous emission dynamics of self-assembled QDs. A reduction of the spontaneous emission lifetime up to $(5.6 \pm 0.3) \times$ was directly measured for dots on resonance with the cavity modes and a lengthening $>2 \times$ off resonance over a band- width $\geqslant 40 \mathrm{~nm}$. From the spectral dependence, a maximum enhancement of $\tau_{0} / \tau_{2}$ up to $19 \pm 4$ was deduced, corresponding to a drastic shorting of the exciton lifetime to only $\sim 50$ ps. This indicates that the single-mode coupling efficiency may become as large as $\beta \sim 97 \%$. Finally, since the numbers reported here are ensemble averages, they may become even larger for an ideally placed dot with a dipole moment aligned perfectly with the cavity field. Therefore, the present results provide significant promise for realization of efficient single-photon emitters based on PC nanocavities.

The authors gratefully acknowledge financial support by Deutsche Forschungsgemeinschaft via Grant No. SFB-631, and J. Zarbakhsh and K. Hingerl from the University of Linz for theoretical input.
*Electronic address: kress@wsi.tu-muenchen.de

${ }^{1}$ E. Yablonovich, Phys. Rev. Lett. 58, 2057 (1987); S. John, ibid. 58, 2486 (1987).

${ }^{2}$ P. Lodahl, A. F. van Driel, A. S. Nikolaev, A. Irman, K. Overgaag, D. Vanmaekelbergh, and W. L. Vos, Nature (London) 430, 654 (2004).

${ }^{3}$ T. F. Krauss and R. M. De La Rue, PIQE 23, 51 (1999).

${ }^{4}$ E. Knill, R. Laflamme, and G. J. Milburn, Nature (London) 409, 46 (2001).

${ }^{5}$ N. Lütkenhaus, Phys. Rev. A 61, 052304 (2000).

${ }^{6}$ T. Yoshie et al., Nature (London) 432, 200 (2004).

${ }^{7}$ H. P. Reithmaier et al., Nature (London) 432, 197 (2004).

${ }^{8}$ P. Michler et al., Nature (London) 406, 968 (2000).

${ }^{9}$ G. S. Solomon, M. Pelton, and Y. Yamamoto, Phys. Rev. Lett. 86, 3903 (2001).

${ }^{10}$ M. Pelton, C. Santori, J. Vučković, B. Zhang, G. S. Solomon, J. Plant, and Y. Yamamoto, Phys. Rev. Lett. 89, 233602 (2002).

${ }^{11}$ J. Hours et al., Appl. Phys. Lett. 82, 2206 (2003).

${ }^{12}$ N. Gisin, G. Ribordy, W. Tittel, and H. Zbinden, Rev. Mod. Phys. 74, 145 (2002).
${ }^{13}$ E. M. Purcell, Phys. Rev. 69, 681 (1946).

${ }^{14}$ L. C. Andreani, G. Panzarini, and J. M. Gérard, Phys. Rev. B 60, 13276 (1999).

${ }^{15}$ J. Vučković and Y. Yamamoto Appl. Phys. Lett. 82, 2374 (2003).

${ }^{16}$ O. Painter, J. Vučković, and A. Scherer, J. Opt. Soc. Am. B 16, 275 (1999)

${ }^{17}$ Y. Akahane, T. Asano, B. Song, and S. Noda, Nature (London) 425, 944 (2003).

${ }^{18}$ The photonic band structure was calculated using the software MiT PHOTONIC BANDS. [S. G. Johnson and J. D. Joannopoulos, Opt. Express 8, 173 (2001)].

${ }^{19}$ J. D. Joannopoulos et al., Photonic Crystals-Moulding the Flow of Light (Princeton University Press, Princeton, NJ, 1995).

${ }^{20}$ The electric field profiles for the two modes are shown as an inset on Fig. 1(c), from which we deduce an effective mode volume of $V_{\text {eff }} \sim 1.5(\lambda / n)^{3}$.

${ }^{21}$ M. Bayer and A. Forchel, Phys. Rev. B 65, 041308(R) (2002).

${ }^{22}$ P. W. Fry et al., Phys. Rev. B 62, 16784 (2000).

${ }^{23}$ J. M. Gérard, B. Sermage, B. Gayral, B. Legrand, E. Costard, and V. Thierry-Mieg, Phys. Rev. Lett. 81, 1110 (1998). 